\title{
Genome Wide Association Mapping for Drought Recovery Trait in Rice (Oryza Sativa L.)
}

\author{
Zaniab Al-Shugeairy ${ }^{1,2}$, Adam H. Price ${ }^{1}$, David Robinson ${ }^{1}$ \\ ${ }^{1}$ Institute of Biological and Environmental Sciences, University of Aberdeen, Aberdeen, UK \\ ${ }^{2}$ Present address: Field Crop Department, College of Agriculture, University of Baghdad, Baghdad, Iraq
}

Email address:

zainab_fa2000@yahoo.com (Z. Al-Shugeairy), david.robinson@abdn.ac.uk (D. Robinson), a.price@abdn.ac.uk (A. H. Price)

\section{To cite this article:}

Zaniab Al-Shugeairy, Adam H. Price, David Robinson. Genome Wide Association Mapping for Drought Recovery Trait in Rice (Oryza Sativa L.). International Journal of Applied Agricultural Sciences. Vol. 1, No. 1, 2015, pp. 11-18. doi: 10.11648/j.ijaas.20150101.12

\begin{abstract}
Rice is the one of the oldest crop cereals in Asia and has been grown since ancient times. In the present study, a rice diversity panel was exposed to drought and drought recovery was scored to identify QTLs and candidate genes related to drought resistance. There are no reports of QTL mapping using Genome wide association mapping for drought recovery has been published. Only one significant association on chromosome 2 for drought recovery with physical position at 24559374 bp was found. positional candidate genes underneath QTL was examined bioinformatically and through the literature revealing several interesting genes which may offer potential for developing drought resistant rice cultivars.
\end{abstract}

Keywords: Mapping, Rice, Drought Recovery, Association

\section{Introduction}

A review by [1] showed that plants which are able to keep green leaf area are better able to recover after drought and provide good yields. [2] noted that leaf drying, often used in field scoring, is the reverse side of the stay green ability and has been shown to be highly linked to relative leaf water content. [1] revealed that leaf retention may be especially significant when stress develops around panicle initiation, since lines with good leaf retention can provide extra assimilate to the developing panicle during subsequent recovery and this eventually results in the production of a greater number of spikelets [3]. Nevertheless, it is difficult to split the green leaf retention from the probable underlying mechanisms of drought resistance and the process of drought recovery. This is not easy in terms of mechanisms, importance or genetic difference as the trait is weakly understood [2]. [4] reported that the ability of the plant to recover after drought was more crucial than drought tolerance. [5] considered drought recovery as the major factor limiting the grain yield productivity for rice under stress. [6] reported that poor recovery from stress could be a main cause of decreased grain yield productivity in rice. Identifying genes that contribute to drought recovery in a quantitative way should allow the utilization of these genes in breeding programmes via marker-assisted selection, and may result in the revealing of genes controlling that trait. [7] reported that 20 markers were used to genotype the 329 $\mathrm{BC}_{2} \mathrm{~F}_{2}$ plants generated from parental lines OM1490 / WAB880-1-38-18-20-P1-HB, OM4495 / IR65195-3B-2-2-22 and OM1490 / WAB881 SG 9. The drought recovery genes on chromosome 9 are positioned between flanked SSR markers, RM201 and RM328 positioned at $0.4 \mathrm{cM}$ and 13.8 $\mathrm{cM}$ respectively.

\section{Material and Methods}

\subsection{Plant Material and Set up the Experiment}

A total of 371 cultivars of the Rice Diversity Panel (http://www.ricediversity.org/) were received from the Susan McCouch, Cornell University and multiplied in Aberdeen in the summer of 2008. A subset of 328 accessions was used for this experiment of those tested, 277 belonged to the rice subpopulations aromatic (9), aus (53), indica (57), temperate japonica (75) or tropical japonica (85). The remaining 48 were classed as admixtures between subpopulations (Figure 1). The layout of this experiment was a randomized complete block design with four replications. The design of blocks was arranged linearly along the length of the box in the NorthSouth orientation that was employed. One soil-filled box with $450 \mathrm{~cm}$ length, $90 \mathrm{~cm}$ width and $40 \mathrm{~cm}$ in depth was prepared. A total of 328 rice diversity panel accessions were sown on $26^{\text {th }}$ August 2011. Supplementary light of $150 \mu \mathrm{mol}$ $\mathrm{m}^{-2} \mathrm{~s}^{-1}$ PAR was supplied for 12 hours a day within 
temperature range from $28-30^{\circ} \mathrm{C}$ and watered with Yoshida's full strength nutrient solution [8]. At 56 days after sowing the water was withheld for 37 days and then returned for 22 days. Theta probes reading were taken regularly.

\subsection{Estimation of Drought Recovery Score}

A modification of the Standard Evaluation System [10] was utilized to score plant recovery. A plant recovery score was taken at 22 days after re-watering according to the percentage of the leaf area that was green (Figure 2). The plants were scored on a scale from 0 to 5 , where score $0,1,2$, 3,4 and 5 represented $100 \%, 90-70 \%, 70-50 \%, 50-30 \%, 30-$ $10 \%$ and $<10 \%$ of the leaf area are recovered green respectively
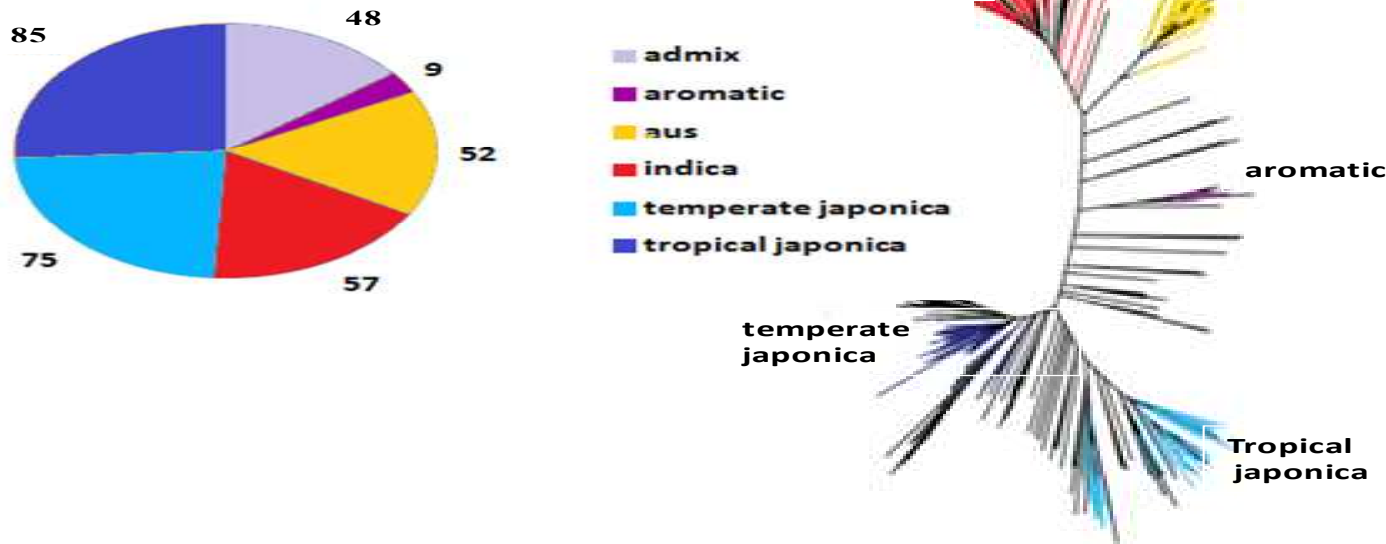

Figure 1. The left pie chart displays the distribution of subpopulations in the 324 O. Sativa samples used in this experiment. The size of the pie chart is proportional to the sample size and colours within each pie chart are demonstrative of the proportion of samples in each subpopulation. The right phylogenetic tree and the branch tips of the tree are coloured according to the subpopulation which are indica, aus, aromatic, tropical japonica and temperate japonica [Source: 9].
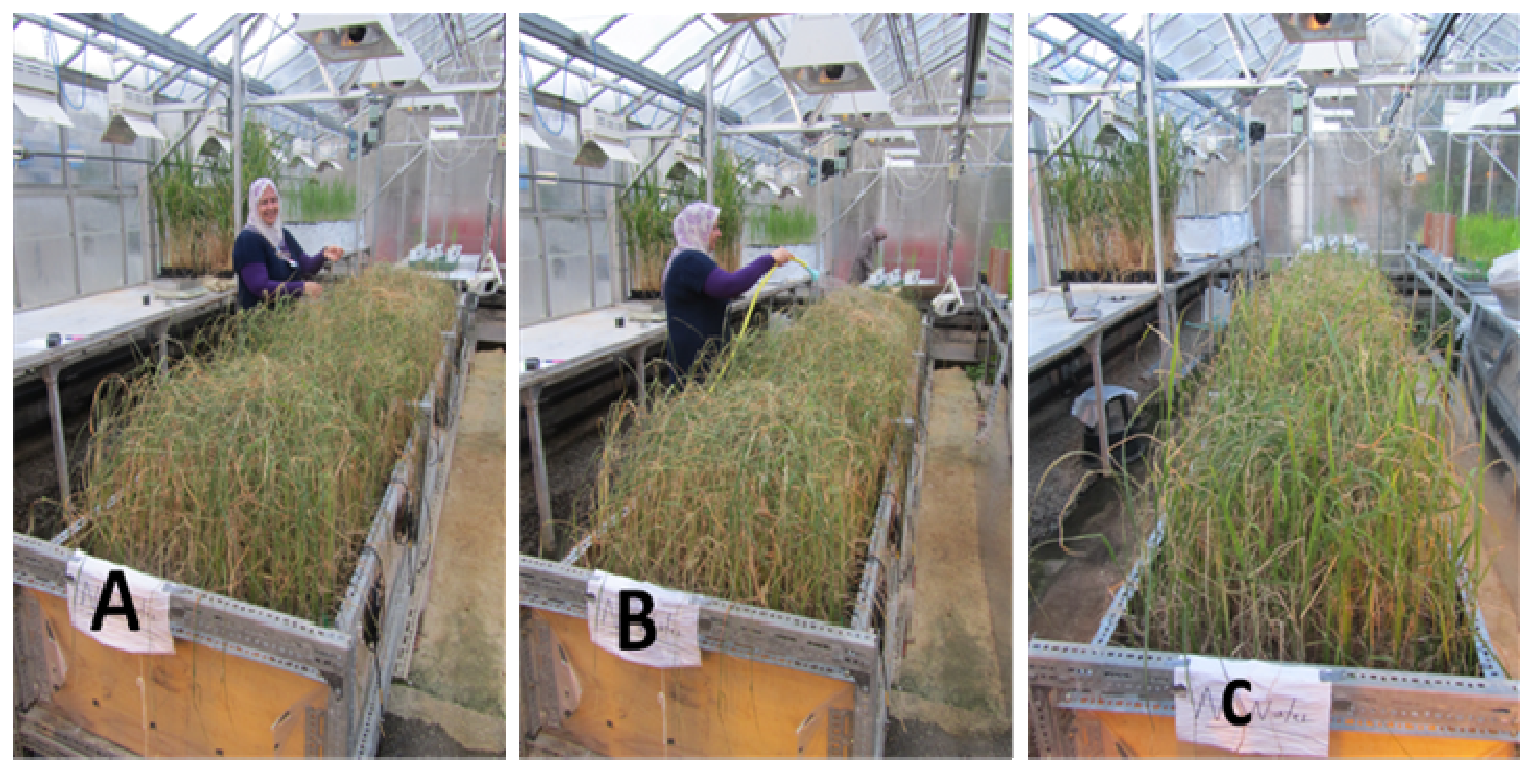

Figure 2. A; Rice plant at 37 days of water stress; B; Re-watering rice plants; $C$; Rice plants at 22 days after re-watering.

\subsection{Association Mapping in Rice}

The rice diversity panel have been genotyped at Cornell University, New York on an Affymetrix genotyping array which comprises of 44,100 SNPs distributed over the rice genome $(380 \mathrm{Mb})$ [11]. [12] reported that with $\sim 1 \mathrm{SNP} / 10 \mathrm{~kb}$ coverage was estimated for this SNP chip. All the analysis for association mapping was performed by Dr. Alexander
Douglas Statistician and Bioinformatician (University of Aberdeen) by using statistical package R. An efficient mixed model analysis (EMMA) taking population structure into account was done on all the genotypes following the methodology reported by [13], which was modified from [14] who developed a novel mixed-model approach to simultaneously account for multiple levels of relatedness detected by random genetic markers. Based on data from a 
maize association mapping project, this approach has excellent type I and type II error rates. In addition, this technique should be readily applicable to a wide range of species and populations, as it estimates population structure based on increasingly available molecular marker data. Separate analysis without population structure was conducted on each of the four most numerous subpopulations separately. This analysis with EMMA plus separate sub-populations is identical to the statistical approach adopted by [11] in the first publication mapping traits using this SNP data on this population. For the result, Dr Douglas provided several files including four pdf files (Histogram, Manhattan mixed, Manhattan naïve and QQ plot). In the present study the mixed models file (text file) that resulted from EMMA was first examined which included a P value, SNP identification, bp position (on the rice genome in base pairs) and chromosomes name for each SNP. Also used was the statistic for the minor allele frequency value which was obtained from previous analysis of other data, and relates to the allele of the SNP that is of lowest frequency within the genotypes. If this proportion was less than $5 \%$, it will mean that SNP is potentially not reliable. For the analysis of the separate subspecies, the aromatic and admixtures were removed from the data set prior to association mapping analyses as there were inadequate numbers of individuals within these groups. The four groups of association populations were analysed for association mapping. The literature suggests that there is no uniform threshold $\mathrm{P}$ value that can be considered in genom wide association mapping [11]. Here, QTLs were considered reportable if they had multiple close (within $200 \mathrm{~kb}$ ) SNPs with low P values (below 0.0001) and where at least some of these SNPs did not have minor allele frequencies below $5 \%$.

\subsection{Candidate Gene Compilation}

Based on the approach of [11], genes situated approximately $200 \mathrm{~kb}$ around associations (excluding transposons) were considered positional candidates (assuming LD of $200 \mathrm{~kb}$ ). Therefore, lists of genes within this region were collected using the rice Pseudomolecule version 6 from the Rice Genome Annotation Project. In order to gather more information about candidate genes, the expression pattern of each was assessed bioinformatically using the rice expression profile database (RiceXPro) http://ricexpro.dna.affrc.go.jp/ after converting Rice Genome Annotation Project (RGAP) names to International Rice Genome Sequencing Project (IRGSP) names at http://rapdb.dna.affrc.go.jp/tools/converter/run. In addition, the candidate genes with clear expression in roots were investigated further in the literature to determine whether they are related to cell expansion or root elongation in other studies which would make them particulary good candidate genes.

\subsection{Statistical and Bioinformatic Analysis}

Minitab version 15 was use to analyse the data. Two-way ANOVA with factors genotype and block was utilized. The data were corrected for the block effect and also for normality by using base $\log _{10}$. The significance of differences between the cultivars in the leaf rolling score and drought recovery score were tested using one way ANOVA. The association mapping analysis was done using efficient mixed model analysis (EMMA).Trait-marker associations were considered reliable with $\mathrm{P}$ values below 0.0001 . The significant SNPs were tested for minor allele frequencies, with values above $5 \%$ considered to be dependable. Candidate genes were selected as positional candidates when they were located with $200 \mathrm{~kb}$ of the QTL identified.All the candidate genes have been tested for full-length cDNA (fl cDNA) and expressed sequence tag (EST) [15] in order to distinguish between genes and likely psuedogenes. The RiceXPro database http://ricexpro.dna.affrc.go.jp/) [16] was used to test gene expression in plant leaves. In addition, the candidate genes with significant expression were examined for whether they are linked to leaf rolling or drought recovery or even to cell expansion through literature searching in order to reduce the number of candidate genes.

\section{Results and Discussion}

\subsection{Soil Water Content}

The soil water content at $15 \mathrm{~cm}$ depth was maintained above $20 \%$ while that at $30 \mathrm{~cm}$ was about $30 \%$ until 56 days after sowing (Figure 3). Theta probe readings of both depths 15 and $30 \mathrm{~cm}$ dropped steadily until both reached $7 \%$ at day 99 after sowing. After the rice plants were re-watered again, the theta probe reading increased sharply

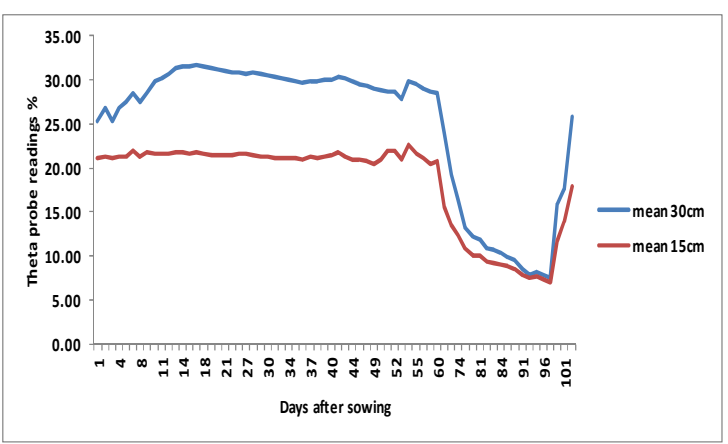

Figure 3. Mean of theta probe readings at two different depths $(15 \mathrm{~cm}$ with red and $30 \mathrm{~cm}$ with blue) throughout the experiment.

\subsection{Drought Recovery Score}

Drought recovery score was assessed at 22 days after irrigating. One-way ANOVA showed that the differences in drought recovery score between the cultivars was highly significant $\left(\mathrm{F}=5.89, \mathrm{P}=0.001, \mathrm{R}^{2}=54.73\right)$. Italica Carolina, KAMENOO, Kihogo, Kon Suito, Kon Suito, M-202, Nucleoryza, SUNG LIAO 2 and YRL-1 had the highest scores for drought recovery indicating that they were least able to recover, while Halwa Gose Red, ARC 10376, DEE GEO WOO GEN, Khao Tot Long 227, SATHI, IR 36, TAICHUNG NATIVE 1, Tchibanga and DA16 had the lowest scores [15] (Figure 4). There was a great and 
significant variation in score of drought recovery in the rice population supported by one-way ANOVA (F 5.89, $\mathrm{P}=0.008$, $\mathrm{R}^{2}=53.8 \%$ ); the temperate japonica group had the highest mean (4.17) while indica had the lowest (2.88) (Figure 5).

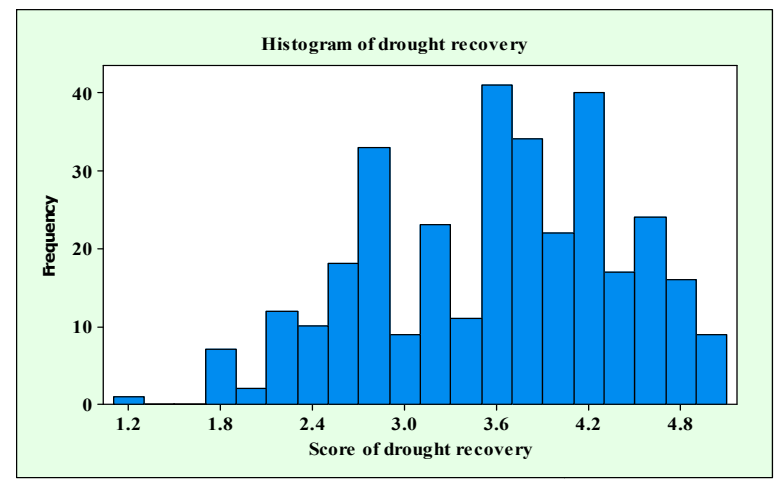

Figure 4. Histogram of drought recovery score for different rice cultivars at 22 days after re-watering.

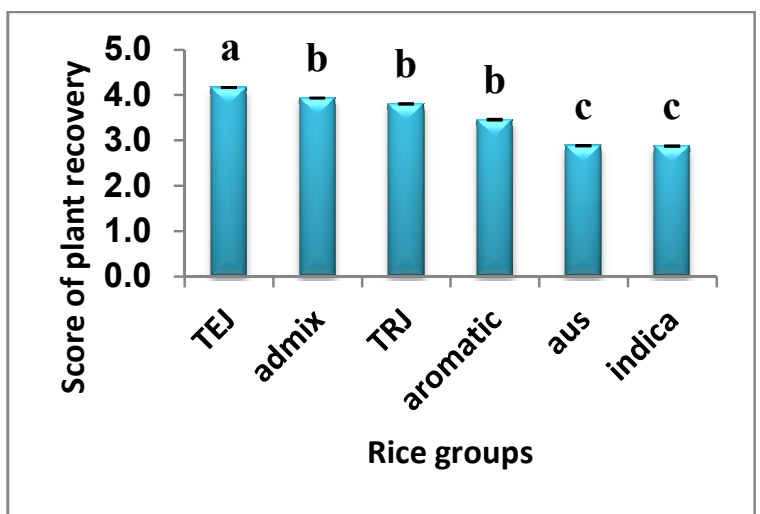

Figure 5. Mean score of drought recovery for rice subpopulations [aromatic $(n=9)$, aus $(n=53)$, indica $(n=57)$, temperate japonica (TEJ) $(n=75)$, tropical japonica (TRJ) $(n=85)$ and admix $(n=48)]$ with standard error bars. Bars that do not share a letter are significantly different.

Figure 6 presents the cumulative distributions of $P$ values in a genome-wide scan for plant drought recovery at 22 days after re-watering again showing the value of the mixed model in controlling false positive associations. Association analysis of the drought recovery score is presented graphically in Figure 7. Use of EMMA revealed that only one SNP is significantly associated with drought recovery using a threashold value from the association analysis of under $0.0001\left(-\log _{10} \mathrm{P}=4\right.$ ) (Figure 8, Table 1). The most significant SNP association was EMMA 2.7. The minor allele frequency is 0.27 , which indicates that this association is reliable. A total of 45 genes had been detected within $200 \mathrm{~kb}$ of this SNP [15]. From association analysis for individual subpopulations indica, aus, temperate japonica and tropical japonica a total of 57, 28, 18 and 15 respectively, significant SNPs associated with drought recovery score were detected (Figure 7). In the present study only those SNPs detected in the mixed model have been taken forward for listing candidate genes. Two studies have reported the presence of QTLs for drought-related traits on chromosome 2 where the QTL is detected in this experiment. A study mapping drought resistance by [17] reported that a total of 154 lines from a doubled-haploid population was generated from a cross between CT9993, a Japonica, and IR62266, an Indica, subspecies. A QTL associated with drought resistance was found on chromosome 2 between markers RM263 and R3393 with physical position (25865334 - 28351861) bp. Another study done by [18] who detected a QTL between markers G45 and G39 on chromosome 2 with physical position (22595831- 27034665). These are close to the physical position of EMMA 2.7 at $24559374 \mathrm{bp}$ and may therefore represent the same QTL.

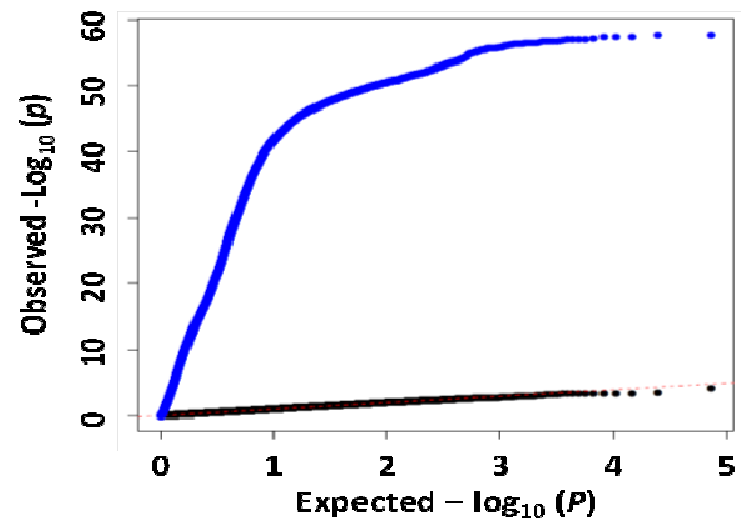

Figure 6. Cumulative distributions of $P$-values in a genome-wide scan for drought recovery at 22 days of re-watering. Blue dotted line is the naïve data plot, Black dotted line is the data corrected for population structure using efficient mixed-model association (EMMA). The red dotted line is a $y=x$ plot.

Table 1. Twenty one associated SNPs with genome-wide significance for drought recovery.

\begin{tabular}{|c|c|c|c|c|c|}
\hline \multicolumn{6}{|l|}{ Association } \\
\hline name & Chromosome & SNP id & Position (bp) & p value & $-\log P$ \\
\hline EMMA1 & 1 & id1002759 & 3369209 & 0.000388 & 3.411 \\
\hline EMMA2.1 & 2 & wd2001280 & 14231552 & 0.000705 & 3.152 \\
\hline EMMA2.2 & 2 & id2006023 & 14651875 & 0.00096 & 3.018 \\
\hline EMMA2.3 & 2 & id2006513 & 16125288 & 0.000424 & 3.373 \\
\hline EMMA2.4 & 2 & id 2006523 & 16161784 & 0.000423 & 3.374 \\
\hline EMMA2.5 & 2 & id2006538 & 16201982 & 0.000414 & 3.383 \\
\hline EMMA2.7 & 2 & id2010408 & 24559374 & $6.88 \mathrm{E}-05$ & 4.163 \\
\hline EMMA2.8 & 2 & id2010581 & 24708094 & 0.000477 & 3.322 \\
\hline EMMA3 & 3 & id3003983 & 7342723 & 0.000925 & 3.034 \\
\hline EMMA4.1 & 4 & id4009537 & 28684807 & 0.000982 & 3.008 \\
\hline EMMA4.2 & 4 & $\mathrm{id} 4010382$ & 30502664 & 0.000548 & 3.261 \\
\hline
\end{tabular}




\begin{tabular}{llllll}
\hline Association & & & & \\
\hline name & Chromosome & SNP id & Position (bp) & p value \\
\hline EMMA4.3 & 4 & id4010399 & 30523167 & 0.000615 & -log P \\
EMMA4.4 & 4 & id4010527 & 31052890 & 0.000644 & 3.211 \\
EMMA6 & 6 & id6001063 & 1472103 & 0.000402 & 3.191 \\
EMMA8.1 & 8 & id8002147 & 6141741 & 0.000418 & 3.379 \\
EMMA8.2 & 8 & id8003182 & 10078371 & 0.000734 & 3.135 \\
EMMA9 & 9 & id9006979 & 19382477 & 0.0004 & 3.398 \\
EMMA10.1 & 10 & id10004316 & 15768637 & 0.000275 & 3.561 \\
EMMA10.2 & 10 & id10005235 & 17866346 & 0.000636 & 3.196 \\
EMMA12 & 12 & id12005910 & 17564316 & 0.000443 & 3.353 \\
\hline
\end{tabular}

The highly significant associations are in bold.

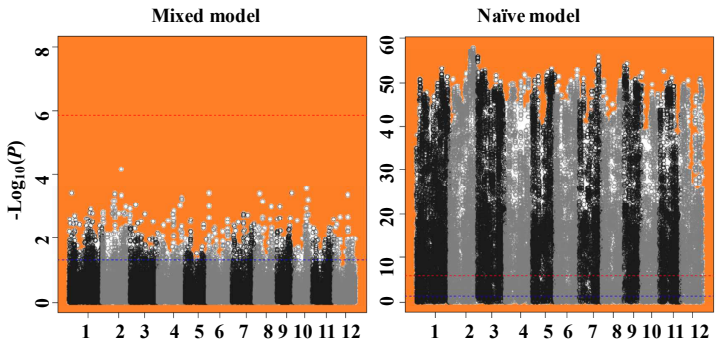

Chromosomes

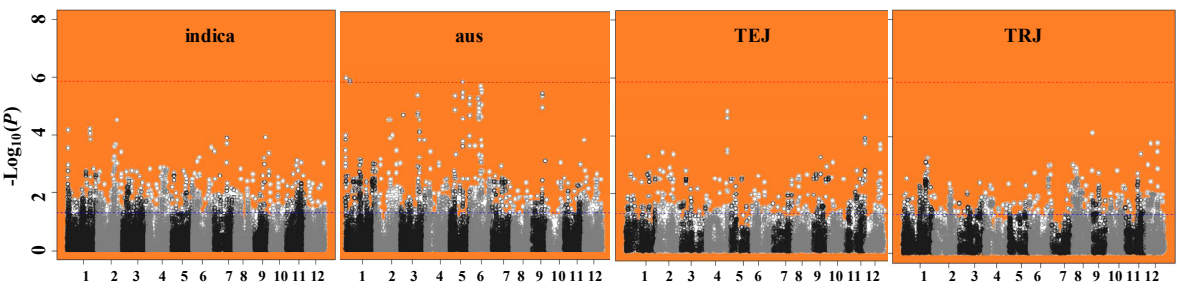

Chromosomes

Figure 7. Genome-wide P-values from the mixed, naïve, and subpopulation method. $x$ axis shows the SNPs along each chromosome; $y$ axis is the - $\log _{10}(P-$ value) for the association.

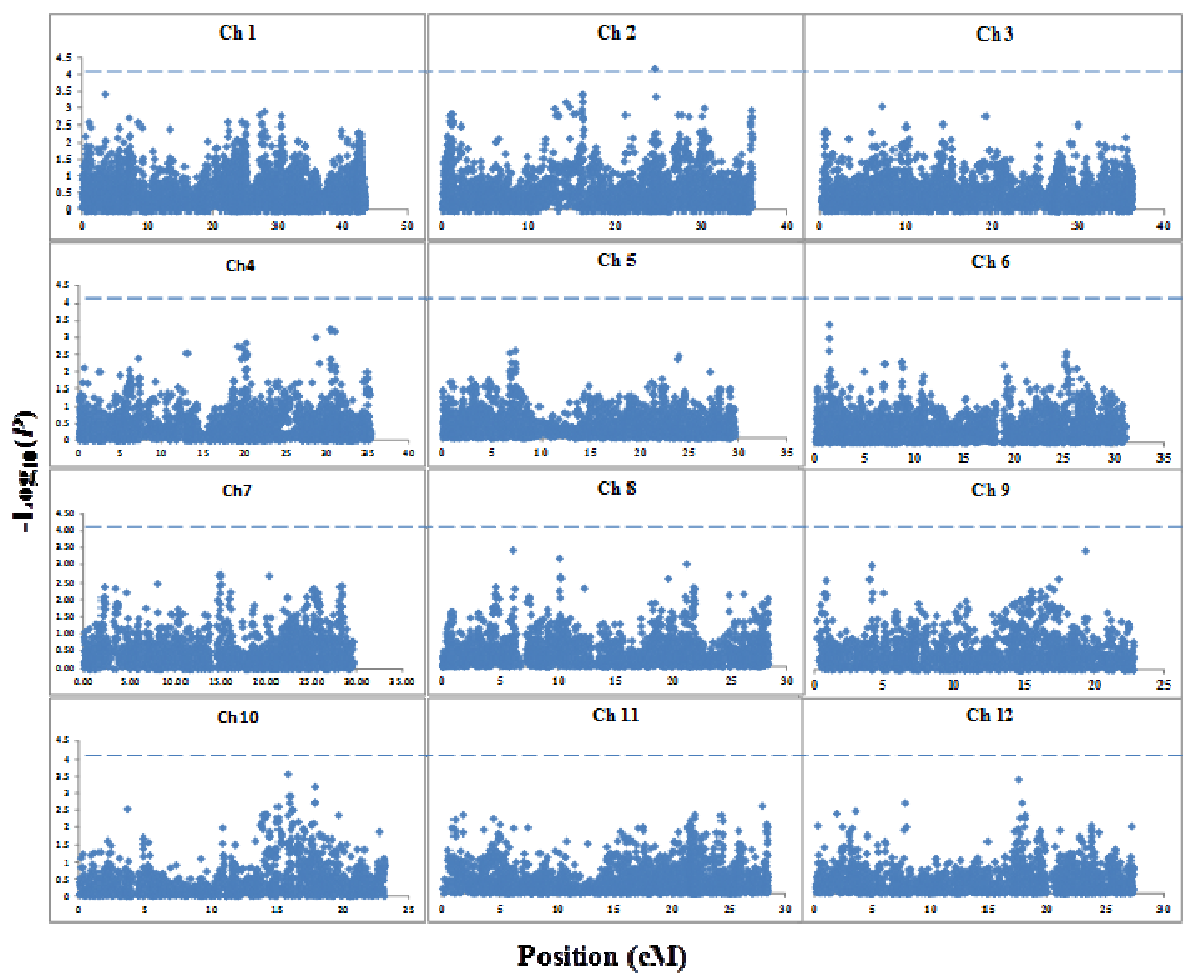

Figure 8. Genome-wide associations of drought recovery score. Data corrected for population structure by efficient mixed-model association. Dashed line shows threshold at $-\log _{10}(P$-value $)=4$. 
By assessing leaf recovery in 328 accessions it was possible to show that there was significant variation across cultivars and subgroups probably reflecting differences in the degree of dehydration experienced or physiological and molecular reactions to cellular water shortage. [19] showed that recovery after a severe drought was a two-stage process. A first stage occurs during the first days upon re-watering, and consists basically in leaf re-watering and stomata reopening. A second stage lasted several days and requires de novo synthesis of photosynthetic proteins.

In the the current study it has been shown that the drought recovery takes place at after day 22 of re-watering while [20], who worked on the responses of Populus euphratica Oliv. plants to soil water deficit reported that plant recovery after drought stress required 10 days after re-irrigating. These differences in time required for drought recovery may be due to differences in plant physiological and biochemical processes between plant species over the drought recovery period. [21] reported that, in several species, restricted recovery of leaf specific hydraulic conductivity is caused by down regulation of stomatal conductance after re-irrigating while another interpretation for drought recovery was given by [22] who showed that aquaporins have a dominant role in the regulation of dynamic variation in hydraulic conductance of leaves. In addition to this, [23] reported that electrical rather than hydraulic signals may have a major role in regulating stomatal re-opening after drought stress in maize. Grames and his colleagues postulated that after serious drought stress, the important limiting factor for photosynthetic recovery is the slowly reversible mesophyll conductance to $\mathrm{CO}_{2}$ as has been shown in a number of Mediterranean species belonging to different growth forms and functional groups.

Therefore, the significant differences between the rice cultivars observed in this study for drought recovery may reflect the effects of drought on photosynthesis, ranging from the restriction on $\mathrm{CO}_{2}$ diffusion into the chloroplast, via limitations on stomatal opening mediated by shoot- and rootgenerated hormones, and on the mesophyll transport of $\mathrm{CO}_{2}$, to alterations in leaf photochemistry and carbon metabolism [24]. These effects vary according to the intensity and duration of the stress as well as with the age of the leaf; older leaves are more affected by drought [24]. In some cultivars a sustained down-regulation of stomatal conductance after reirrigation imposes a substantial limitation to photosynthetic recovery, at the time that it increases the intrinsic water-use efficiency [20].

The result in the present study confirmed that there was noticeable subpopulation structure among these accessions of rice (Figure 5). The Indica subspecies (indica and aus subpopulations) had significantly higher leaf recovery score than other subpopulations, which might be due to differences in stomatal aperture. [25] showed that stomatal apertures of Indicas were higher than Japonicas and that this factor causes a difference of leaf conductance between these rice sub groups. Stomatal conductance could be measured on these plants if the experiment was conducted again, although it would certainly be a major practical challenge. Also significant is the observation that osmotic adjustment is known to be more prominent in Indicas than Japonicas [26] which might mean that the former have a higher water status at the end of the drought. It would be useful to test water potential at the end of the drought or osmotic adjustment in the panel, but these are very labour intensive traits to measure.

Analysis of association mapping revealed that a total of three genes were identified as good candidates for the QTLs for drought recovery detected here. Based on position and expression in different tissues, 28 candidate genes were expressed in leaf and other plant tissues, 16 genes did not show any expression and one gene was expressed in other plant tissues but not in leaf tissue [15]. From these, three stand out after investigating gene function in the literature. This is summarised below.

MYB family transcription factor (LOC_Os02g40530), is a member of a large 198 gene family from an examination of the complete Arabidopsis genome sequence. Of those, 126 are R2R3-MYB, 5 are R1R2R3-MYB, 64 are MYB-related, and 3 atypical MYB genes [27]. This gene had expression intensity in leaf tissue that reaches approximately $500 \mathrm{Cy} 3$. MYB proteins are fundamental factors in regulatory networks governing development, metabolism and reactions to biotic and abiotic stresses. The first gene encoding a transcription factor in plants (COLORED1 (C1) locus) was found to encode a MYB domain protein necessary for the synthesis of anthocyanins in the aleurone of maize (Zea mays) kernels [28]. AtMYB91/AS1 regulates shoot morphogenesis and leaf patterning through its competitive actions with KNOX proteins [29]. AtMYB60 and AtMYB96 act through the ABA signalling cascade to regulate stomatal movement [30] and drought stress[31]. Thus this gene might have a role in the drought stress response of plants.

Response regulator receiver domain containing protein (LOC_Os02g40510) is considered a good candidate because a link to function in drought stress can be shown. This gene has an expressed intensity in leaf tissue of about $4000 \mathrm{Cy} 3$ [15]. This gene matches AT5G61380.1, which is annotated as APRR1, ATTOC1, PRR1, pseudo-response regulator 1, timing of $\mathrm{CAB}$ expression 1 and TOC1, which is a main gene of the circadian clock that regulates the coordination of gene expression in relation to day/night cycles [32]. This regulation is very important for hormone abscisic acid (ABA) function, which regulates stress signals and is crucial for plant tolarance to adverse environmental conditions, as TOC1 and ABA-related gene overexpressing and mutant plants exhibit altered ABA-mediated resistance to drought circumstances [33].

Being expressed in leaf tissue, Enzyme of the cupin superfamily protein (LOC_Os02g40700) could be considered as another good candidate. Using RiceXPro revealed that the expression intensity of this gene reach approximately 2000 Cy3 in leaves. According to [34], germin and germin-like 
proteins (GLPs) are encoded by a family of genes found in all plants. The GLPs are part of the cupin superfamily of biochemically different proteins. In terms of function, the GLPs are known to be differentially expressed during specific stages of plant growth and development. They are also implicated in the response of plants to abiotic (salt, heat/cold, drought, nutrient and metal) stress [34]. This involvement with the protection of plants from environmental stress of different types has led to massive plant breeding studies that have established links between GLPs and QTLs for stress resistance.

\section{Conclusion}

Improving the drought resistance of high yielding rice (Oryza sativa L.) varieties for areas prone to drought is a goal of rice breeders. Therefore, understanding the mechanisms affecting drought resistance is an important issue for rice breeding. However, the mechanisms underlying drought resistance are complex. Identifying quantitative trait loci (QTLs) which confer drought resistance promises to speed up this goal. In this study differences in drought recovery between rice cultivars has been revealed and QTL identified. These data indicate areas of the rice genome containing genes of potential value in breeding drought resistant rice. These candidate genes are worth further investigation.

\section{References}

[1] Fukai, S and Cooper, M (1995). Development of droughtresistant cultivars using physiomorphological traits in rice. Field crops research, 40: $67-86$.

[2] Price, AH and Courtois, B (1999). Mapping QTLs associated with drought resistance in rice: progress, problems and prospects. Plant Growth Regul, 29: 123 - 133.

[3] Lilley, JM and Fukai, S (1994). Effect of timing and severity of water deficit on four diverse rice cultivars III. Phenological development, crop growth and grain yield. Field Crops Res, 37: $225-234$.

[4] Maji, A (1994). Vegetative stage drought tolerance and agronomic characteristics of Oryza glaberrima accessions. Msc Thesis University of Ibadan, pp156.

[5] De Datta, S; Chang, K and Yoshida, S (1975). Drought Tolerance in upland Rice. ln: Major Research in Upland Rice. IRRI Los Banos Philippines, 205:101 - 116.

[6] Malabuyoc, J; Aragon, E and De Datta, S (1984). Recovery from drought induced desication at vegetative growth stage in direct seeded rain fed rice. Field Crop Research, 10: 105 - 112.

[7] Lang, NT and Buu, BC (2008).fine mapping for drought tolerance in rice (Oryza sativa L.). Omonrice, 16: 9 - 15.

[8] Yoshida S, Forno DA, Cock JH, Gomez KA, (1976). Laboratory manual for physiological studies of rice. Philippines: IRRI; 83.

[9] Zhao, K; Wright, M; Kimball, J; Eizenga, G; mcclun, A; Kovach, M; Tyagi, W; Ali, ML; Tung, CW; Reynolds, A;
Bustamante, C and Mccouch, Sr (2010). Genomic diversity and introgression in $O$. sativa reveal the impact of domestication and breeding on the rice genome. PLOS One 5, e10780.

[10] IRRI, (1975). Standard Evaluation system for Rice. International Rice Research Institute. Los banos, Philippines. pp. 64.

[11] Zhao, K; Tung, CW; Eizenga, GC; Wright, MH; Ali, ML; Price, Ah; Norton, GJ; Islam, MR; Reynolds, A; Mezey, J; McClung, A; Bustamante, C and McCouch, SR (2011). Genome-wide association mapping reveals a rich genetic architecture of complex traits in Oryza sativa. Nature communications, 2: 467.

[12] Tung, CW; Wright, M; Zhao, K; Reynolds, A; Montgomery, J; Tanimoto, G; Barkovich, R; Pirani, A; Eizenga, G; McClung, A; Bustamante, C and McCouch, S (2009). Design and performance of 44,100 SNP genotyping array for rice. Poster presented as part of the Plant and Animal Genomes XVIII conference, San Diego, California, 9-13 January 2010. Abstract published at "http://www.intl-pag.org/", visited on 10 October 2012.

[13] Kang, HM; Zaitlen, NA; Wade, CM; Kirby, A; Heckerman, D; Daly, MJ and Eskin, E (2008). Efficient control of population structure in model organism association mapping. Genetics, 178: 1709 - 1723.

[14] Yu , J and Buckler, ES (2006). Genetic association mapping and genome organization of maize. Curr Opin Biotechnol, $17: 155-160$.

[15] Alshugeairy, Z. (2013). Genetic, Phenomic and Molecular Analysis of Drought Avoidance and Recovery Traits in Rice for the Improvement of Plant Breeding. $\mathrm{PhD}$ thesis. Department of Plant and Soil Science. University of Aberdeen, UK

[16] Sato, Y; Antonio, B; Namiki, N; Takehisa, H; Minami, H; Kamatsuki, K; Sugimoto, K; Shimizu, Y; Hirochika, H and Nagamura, Y (2011). RiceXPro: a platform for monitoring gene expression in japonica rice grown under natural field conditions. Nucleic Acids Research, 39: 1141 - 1148

[17] Zhang, J; Zheng, HG; Aarti, A; Pantuwan, G; Nguyen, TT; Tripathy, JN; Sarial,AK; Robin, S; Babu, RC; Nguyen, BD; Sarkarung, S; Blum ,A and Nguyen, HT (2001 ). Locating genomic regions associated with components of drought resistance in rice: comparative mapping within and across species. Theor Appl Genet, 103: 19 -29.

[18] Khowaja, F; Norton, GJ; Courtois, B and Price, AH ( 2009). Improved resolution in the position of drought-related QTLs in a single mapping population of rice by meta-analysis. http://www.biomedcentral.com/1471-2164/10/276.

[19] Kirschbaum, M (1988). Recovery of photosynthesis from water stress in Eucalyptus pauciflora - a process in two stages. Plant Cell and Environment, 11: 685 - 694.

[20] Bogeat-Triboulot, MB; Brosche, M; Renaut, J; Jouve, L; Le Thiec, D; Fayyaz, P; Vinocur, B; Witters, E; Laukens, K; Teichmann, T; Altman,A; Hausman, J; Polle, A; Kangasjärvi, $\mathrm{J}$ and Dreyer, E (2007). Gradual soil water depletion results in reversible changes of gene expression, protein profiles, ecophysiology, and growth performance in Populus euphratica, a poplar growing in arid regions. Plant Physiology, 143: 876 892. 
[21] Galme's, J; Medrano, H and Flexas, J (2007). Photosynthetic limitations in response to water stress and recovery in Mediterranean plants with different growth forms. New Phytologist, 175: 81 - 93.

[22] Cochard, H; Venisse, J; Barigah, T; Brunel, N; Herbette, S; Guilliot, A; Tyree, M and Sakr, S (2007). Putative role of aquaporins in variable hydraulic conductance of leaves in response to light. Plant Physiology, 143: 122 - 133.

[23] Grams, T; Koziolek, C; Lautner, S; Matyssek, R and Fromm, J (2007). Distinct roles of electric and hydraulic signals on the reaction of leaf gas exchange upon re-irrigation in Zea mays L. Plant Cell and Environment, 30: 79 - 84.

[24] Flexas, J; Bota, J; Loreto, F; Cornic, G and Sharkey, TD (2004). Diffusive and metabolic limitations to photosynthesis under drought and salinity in C3 plants. Plant Biology, 6: 269 -279 .

[25] Maruyama, S and Tajima, K (1990). Leaf conductance in japonica and indica rice varieties. I. Size, frequency and aperture of stomata. Japan J Crop Sci, 59: 801- 808.

[26] Lilley, JM; Ludlow, MM; McCouch, SR and O'Toole, JC (1996). Locating QTL for osmotic adjustment and dehydration tolerance in rice. Journal of Experimental Botany, 47(302): 1427 - 1436.

[27] Yanhui, C; Xiaoyuan, Y; Kun, H; Meihua, L; Jigang, L; Zhaofeng, G; Zhiqiang, L; Yunfei, Z; Xiaoxiao, W; Xiaoming, Q; Yunping, S; Li, Z; Xiaohui, D; Jingchu, L; Xing-Wang, D; Zhangliang, C; Hongya, G and Li-Jia, Q (2006).The MYB transcription factor superfamily of Arabidopsis: expression analysis and phylogenetic comparison with the rice MYB family. Plant Mol Biol, 60(1):107 - 24.
[28] Paz-Ares J, Ghosal D, Wienand U, Peterson PA, Saedler H (1987). The regulatory c1 locus of Zea mays encodes a protein with homology to myb proto-oncogene products and with structural similarities to transcriptional activators. EMBO J $6: 3553-3558$.

[29] Byrne, M.E; Simorowski, J; and Martienssen, RA (2002). ASYMMETRIC LEAVES1 reveals knox gene redundancy in Arabidopsis. Development, 129: 1957 - 1965.

[30] Cominelli, E; Galbiati, M; Vavasseur, A; Conti, L; Sala, T; Vuylsteke, M; Leonhardt, N; Dellaporta, SLand Tonelli, C (2005). A guard-cell-specific MYB transcription factor regulates stomatal movements and plant drought tolerance. Curr Biol, 15:1196 - 1200.

[31] Seo, P and Park, C (2010). MYB96-mediated abscisic acid signals induce pathogen resistance response by promoting salicylic acid biosynthesis in Arabidopsis. The New phy, 186: 471- 483.

[32] Matsushika, A; Makino, S; Kojima, M and Mizuno, T (2000). Circadian Waves of Expression of the APRR1/TOC1 Family of Pseudo-Response Regulators in Arabidopsis thaliana: Insight into the Plant Circadian Clock. Plant Cell Physiol, 41 (9): 1002 - 1012.

[33] Castells, E; Portolés, S; Huang, W and Mas, P (2010). A functional connection between the clock component TOC1 and abscisic acid signaling pathways. Plant Signal Behav, 5(4):409 - 11.

[34] Dunwell, J; Gibbings,G; T Mahmood, T and Naqvi, S (2008). Germin and Germin-like Proteins: Evolution, Structure, and Function. Critical Reviews in Plant Sciences, 27 (5):342 - 375. 